\title{
Uplifting the baryonic branch: a test for backreacting anti-D3-branes
}

\author{
Anatoly Dymarsky ${ }^{a}$ and Stefano Massai ${ }^{b, c}$ \\ ${ }^{a}$ Department of Applied Mathematics and Theoretical Physics, University of Cambridge, \\ Wilberforce Road, Cambridge, CBз 0WA, U.K. \\ ${ }^{b}$ Institut de Physique Théorique, CEA Saclay, \\ Orme des Merisiers, F-91191 Gif-sur-Yvette, France \\ ${ }^{c}$ Arnold Sommerfeld Center for Theoretical Physics, \\ Theresienstr. 37, 80333 München, Germany \\ E-mail: dymarsky@damtp.cam.ac.uk, stefano.massai@lmu.de
}

ABSTRACT: Placing D3 or anti-D3-branes at the tip of the Klebanov-Strassler background results in uplifting the baryonic branch of the moduli space of the dual field theory. In this paper we derive a mass formula for the scalar particle associated with the motion along the baryonic branch, from both open and closed string points of view. We show that both methods give the same mass at linear order in number of (anti)D3-branes, thus providing a comprehensive check for the recently found linearized supergravity solution describing backreacting anti-D3-branes at the tip.

KEYwords: Supersymmetry Breaking, AdS-CFT Correspondence

ArXiv ePrint: 1310.0015 


\section{Contents}

$\begin{array}{llr}1 & \text { Introduction } & 1\end{array}$

2 Baryonic branch uplift $\quad 3$

2.1 Baryonic branch from supergravity 3

2.2 Boundary conditions: a puzzle and a resolution 4

$\begin{array}{lll}2.3 & \text { The baryonic branch uplift from supergravity } & 7\end{array}$

3 Gaugino mass perturbation $\quad 10$

4 Uplift of baryonic branch by (anti)D3-branes 12

$\begin{array}{lll}4.1 & \text { Uplift from supergravity } & 12\end{array}$

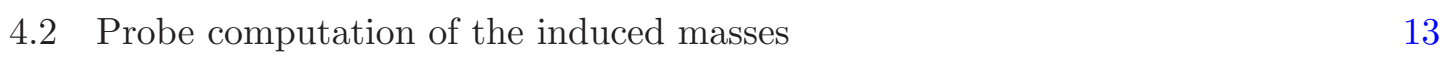

$\begin{array}{lll}5 & \text { Discussion } & 15\end{array}$

$\begin{array}{ll}\text { A Derivation of } V_{\tilde{z}} & 16\end{array}$

$\begin{array}{ll}\text { B Particulars of numerical integration } & 17\end{array}$

\section{Introduction}

For the last thirteen years Klebanov-Strassler (KS) background [1] enjoys the role of a default playground to test various ideas of gauge/gravity duality, whenever maximally supersymmetric $\mathcal{N}=4 \mathrm{YM}$ theory wouldn't suffice. In particular in $2001 \mathrm{Kachru}$, Pearson and Verlinde suggested that placing anti-D3-branes at the tip of the KS geometry would lead, through the backreaction of anti-D3s, to a holographic background dual to a SUSYbreaking metastable state in the dual field theory [2]. This so-called KPV metastable state is peculiar from the field theory point of view. Conceivably because of the absence of a small dimensionless parameter characterizing this state, so far it was out of reach for all known field theoretic techniques. At the same time the corresponding hypothetical background is a key ingredient in the most explicit scenario proposed to date that achieves a four-dimensional de-Sitter in a String Theory compactification [3]. Since the holographic background in question was never constructed explicitly, presumably because of its complexity, the fate of the String Theory landscape of the $d S$ compactifications is currently resting on the original probe D-brane calculation [2]. In light of the importance of the question it is only natural to wish to obtain the backreacted supergravity background explicitly. This ambitious task was first seriously examined in [4] where the authors put forward all necessary ingredients to fully investigate $\mathrm{SU}(2) \times \mathrm{SU}(2) \times \mathbb{Z}_{2}$ invariant sector 
of the background in question at the linear order in number of anti-D3-branes (the earlier attempts $[5,6]$ used various approximations which hamper their ability to determine the desired background simultaneously near and far away from the anti-D3-branes). The original work [4] revealed complexity of the problem arising already at the linearized level, including subtlety of formulating boundary conditions that would correspond to the desired KPV state. This work continued in [7-9] until the $\mathrm{SU}(2) \times \mathrm{SU}(2)$ invariant mode of the corresponding linearized solution was obtained in the most possible explicit form.

Now, once the linearized solution is found, it is very desirable to submit it to all possible self-consistency tests and checks, especially because many technical details associated with formulating boundary conditions etc. were at the time a subject of a heated debate. A simplest consistency check comes from studying interaction potential between a number of anti-D3-branes sitting at the tip of the KS geometry and a stack of D3-branes located some finite distance away. At the linearized order in number of both D3s and anti-D3s there are two ways to proceed. One is to treat D3s as sources backreacting on the geometry while anti-D3s would be probes [10]. Another way is to treat D3s as probes in the (linearized) geometry created by the backreacting anti-D3s. Both calculations yield the same result [7, 11], but unfortunately this test is only sensitive to the IR boundary condition for just one (out of many) supergravity mode. Hence this hardly passes as a very comprehensive check.

Another check of the linearized solution for anti-D3-branes comes from comparing vacuum energy of the dual state in field theory (the ADM mass in supergravity) with the corresponding probe calculation. This check is sensitive to both IR and UV behavior of the solution but certainly does not involve every single part of it; it is possible to show that the solution in question passes this check, without actually obtaining all parts of the solution explicitly [7]. In this paper we propose and perform yet another check which is sensitive to all parts of the solution in question.

The idea of our check is to study the effect of anti-D3-branes on the so-called baryonic branch of moduli space from both open and closed string points of view. Before we go into details, let us take a quick detour and remind the reader basic facts about the KS solution which are relevant to what follows. The field theory dual to the KS background is a particular $\mathcal{N}=1 \mathrm{SU}(N+M) \times \mathrm{SU}(N)$ SYM with $N$ divisible by $M$. This theory admits a so-called baryonic branch of moduli space and in fact the KS background is dual to a particular $\mathbb{Z}_{2}$ symmetric vacuum located at the locus of the baryonic branch. There is a whole one-dimensional family of distinctive vacua in field theory and there is a family of supergravity solutions dual to them in a holographic sense [12-15]. The KS background is only a particular solution from this family. Adding D3 or anti-D3-branes to the KS geometry changes $N$ and as a result the baryonic branch gets uplifted i.e. the massless scalar particle associated with the motion along the branch becomes massive. The corresponding emergent potential near the origin of the branch can be calculated with ease up to linear order in number of (anti)D3-branes using probe approximation [16]. The idea of this paper is to perform the same calculation using closed string channel i.e. by taking the backreacted solution describing (anti)D3-branes and studying the mass of the deformation along the direction in the field space associated with the motion along the baryonic branch. As advertised in the abstract the linearized solution describing the KPV state passes this test with the flying colors. 
Before we proceed with the technicalities let us point out that the machinery developed in the paper to study the uplift of the baryonic branch due to (anti)D3-branes is universal in a sense that it can be used to study the uplift due to any $\mathbb{Z}_{2}$-invariant small perturbation of the KS background. ${ }^{1}$ To better explain the logic of our calculation we in fact start by considering the uplift of the baryonic branch associated with perturbing the original $\mathcal{N}=1$ theory by SUSY-breaking gaugino masses. A naive bulk calculation indicates a non-trivial uplift in this case which is in contradiction with the field theory result. A more careful consideration reveals a subtlety in a way UV boundary conditions should be imposed. A spin-off result of this paper is a rigorous procedure to fix UV boundary conditions based on the asymptotic behavior of the D5-charge.

This paper is organized as follows. In section 2 we develop the machinery necessary to calculate the uplift of the baryonic branch at the origin (mass of the scalar associated with motion along the branch) for any $\mathrm{SU}(2) \times \mathrm{SU}(2) \times \mathbb{Z}_{2}$ perturbation of the KS background. In section 3 we apply these results to calculate the uplift due to infinitesimally small gaugino masses. In section 4 we calculate the uplift for (anti)D3-branes placed at the tip from both open and closed string points of view and compare the results thus testing the linearized solution describing the KPV state. We discuss our findings in section 5. Technical details are delegated to the appendices.

\section{Baryonic branch uplift}

In this section we develop the machinery necessary to calculate the uplift of the baryonic branch near the origin, due to arbitrary $\mathbb{Z}_{2}$-even perturbation.

\subsection{Baryonic branch from supergravity}

As we briefly mentioned in the introduction the KS solution is only one particular point from the so-called BGMPZ family of supergravity solutions [15] which share the same leading UV-asymptotic and hence correspond to different vacua of the same filed theory. The vev $U=\langle\hat{U}\rangle$ of the bottom component of the baryonic $\mathrm{U}(1)$ current

$$
\hat{U}=\operatorname{Tr}\left(|A|^{2}-|B|^{2}\right),
$$

is usually chosen to be the parameter along the branch. The point $U=0$ corresponds to the $\mathrm{KS}$ solution. There is a $\mathbb{Z}_{2}$ symmetry acting as $U \rightarrow-U$ leaving the KS solution invariant (the geometric meaning of this symmetry is discussed in [17]). From the supergravity point of view infinitesimal motion along the branch near the origin $U=0$ is associated with the $\mathbb{Z}_{2}$-odd linear deformation of the KS solution that satisfies certain boundary conditions in the UV. The corresponding supergravity mode $\tilde{z}(\tau)$ was first found by Gubser, Herzog and Klebanov $(\mathrm{GHK})$ in $[13,14]$. It satisfies a Schrödinger-type equation in the radial direction of the conifold $\tau$

$$
-\tilde{z}^{\prime \prime}+V_{\tilde{z}}(\tau) \tilde{z}=0
$$

\footnotetext{
${ }^{1}$ This is more general than small perturbations (by various operators) of the dual theory as here we also assume one can add small number of D-branes which, strictly speaking, change the dual field theory in a non-continuous way.
} 
Zero eigenvalue in (2.2) is due to the fact that motion along the baryonic branch costs no energy i.e. the branch is flat.

The particular form of $V_{\tilde{z}}$ in (2.2) (see section 2.3) leads to the following general solution for $\tilde{z}$ in the UV region $\tau \rightarrow \infty$

$$
\tilde{z}=\alpha\left(\tau-\tau_{0}\right)+\beta,
$$

where $\alpha$ and $\beta$ are some constants, and the constant $\tau_{0}$ is not physical as it always can be absorbed into $\beta$. Taking into account that $\tau \sim \log r$ where $r$ is the "AdS" radius of the conifold we find that (2.3) is consistent with the dimension 2 of the operator $\hat{U}=\operatorname{Tr}\left(|A|^{2}-\right.$ $\left.|B|^{2}\right)$. Usually $\alpha$ would correspond to the coupling and $\beta$ to the vev of $\hat{U}$. But in this particular case these roles happen to be reversed: $\alpha$ is the vev and $\beta$ is the coupling $[13,14]$. Although this is somewhat unusual there is no contradiction with the AdS/CFT dictionary because for the dimension 2 operator both interpretations are valid [18]. The asymptotic of the GHK mode associated with the motion along the branch is $\tilde{z} \sim(\tau-1)$ which means the vev $\alpha \sim U$ changes while the coupling $\beta$ remains zero.

\subsection{Boundary conditions: a puzzle and a resolution}

What happens if we deform the KS background by introducing some small $\mathbb{Z}_{2}$-even perturbation? Unless we are lucky this will result in the baryonic branch uplift i.e. a non-trivial potential $V(U)$ emerging along the branch

$$
V(U)=V(0)+m_{U}^{2} U^{2}+\mathcal{O}\left(U^{4}\right) .
$$

The mass $m_{U}^{2}$ will appear as an eigenvalue in the equation (2.2) as a response to a new effective potential $V_{\tilde{z}}(\tau)$. Below we will develop a machinery to calculate new $V_{\tilde{z}}$ for any $\mathbb{Z}_{2}$-even perturbation of KS and hence the problem of calculating $m_{U}^{2}$ will reduce to some trivial numerics.

Our logic is clear and seems to be completely infallible but in fact we will immediately run into a puzzle if we consider D3-branes as a perturbation. One can be absolutely certain that the D3-branes sitting anywhere on the conifold create a potential of the form (2.4) with some non-zero $m_{U}^{2}[16]$. At the same time direct calculation or reasoning based on the particulars of the GHK geometry ${ }^{2}$ reveal that $V_{\tilde{z}}$ does not change and hence the same GHK mode still solves the equation (2.2) with zero eigenvalue. The only possible interpretation of this would be that D3-branes do not uplift the branch, at least at the lowest order in

\footnotetext{
${ }^{2}$ The deformed conifold, the geometry behind the KS solution, is Ricci-flat. In fact the metric of the GHK perturbation is also Ricci-flat, as can be deduced from the constant dilaton of the GHK solution (also see [19] for a detailed discussion). For such a background the supergravity equations factorize [20]: the only remaining equation is one for the warp-factor $h$. Presence of D3-branes affects the warp-factor through the equation $\nabla^{2} \delta h=\sum \delta_{D 3}$. At the linear order in $U$ the $\mathbb{Z}_{2}$-odd GHK perturbation of the unwarped metric can not affect $\nabla^{2}$ because it is $\mathbb{Z}_{2}$-even. Hence the GHK background with the new warp-factor solves the supergravity equations of motion in the presence of D3-branes up to quadratic order in $U$. Alternatively, one can see that presence of D3-branes does not affect $V_{\tilde{z}}$ in $(2.2)$ because the only part of the background affected by the D3-branes is the warp-factor, but the explicit expression for $V_{\tilde{z}}$ (it is derived later in the text) is warp-factor independent.
} 
$U$. This puzzling contradiction is something we have to resolve before turning to a more complicated case of anti-D3-branes.

It is tempting to attribute the mismatch between the probe and SUGRA calculations to a singular nature of the supergravity background whenever D3-brane are present. This would certainly remove the puzzle but a detailed consideration shows that the suspected singularity of $\delta h$ is not invalidating supergravity-based calculation. Instead of providing a detailed justification of this fact, let us instead sharpen the puzzle by considering another confusing example when the supergravity background remains smooth and weakly curved. Let us perturb the dual gauge theory by the infinitesimally small gaugino mass $m \lambda^{2}$ by giving a non-zero value to the top component of the gauge coupling superfield $\tau=\frac{i}{g^{2}}+\cdots+\theta^{2} m$

$$
\mathcal{L}=\int d^{2} \theta \tau W_{\alpha}^{2}
$$

Here $W_{\alpha}$ is a chiral superfield. Presence of the top component in $\tau$ breaks supersymmetry, but at the linear order in $m$ holomorphy implies that the usual expression for the lowenergy superpotential still holds $W \sim e^{i \tau}$. The resulting potential $V=\int d^{2} \theta W$ acquires the following linear-in- $m$ contribution (to derive this formula one can think of $m$ as a vev of some auxiliary chiral superfield)

$$
\delta V=m \Lambda^{3}+\text { c.c. }
$$

The internal scale of the field theory $\Lambda^{3}=\left\langle\lambda^{2}\right\rangle$ does not depend on the choice of vacuum along the baryonic branch [16] and therefore (2.6) contributes to $V(0)$ in (2.4), but not to $m_{U}^{2}$ which remains zero at linear order in $m$. One can arrive at the same conclusion by noticing that $m$ has $\mathrm{U}(1)_{R}$ charge -3 while $m_{U}^{2}$ is $\mathrm{U}(1)_{R}$ invariant. Hence the only possible dependence on $m$ at linear level could be $m_{U}^{2} \sim m \Lambda^{2} / \Lambda^{*}+$ c.c. which is not allowed because of the complex conjugate $\Lambda^{*}$ in the denominator.

In the discussion above we were sloppy and disregarded the fact that the dual gauge theory has not one but two gauge groups and correspondingly two pairs of gauginos. But exactly the same logic shows that $m_{U}^{2}$ can not depend on neither of two gaugino masses $m_{i}, i=1,2$.

What do we see on the supergravity side? Two linearized supergravity backgrounds dual to the KS theory perturbed by the infinitesimal gaugino masses were explicitly found in $[4,7,21]$. If we calculate new $\delta V_{z}$ we will see that it is non-zero and correspondingly there is no reason to believe new (2.2) admits zero eigenvalue solution any more. The new eigenvalue at linear order in $m_{i}$ is thus

$$
m_{U}^{2}=a_{i} m_{i}
$$

where $a_{i}$ are some numerical coefficients. One can clearly combine $m_{1}, m_{2}$ such that (2.7) vanishes but in a general case the gaugino masses induce a non-vanishing uplift of the baryonic branch. Thus we again run into a contradiction: the supergravity calculation does not match the dual field theory analysis.

We believe by this point we have intrigued our reader enough. The situation seems to be very puzzling: in one case the field theory suggests the uplift should be zero, but 
supergravity produces a non-vanishing answer. In the other case probe calculation gives a non-zero result, but supergravity suggests the uplift is not there.

The resolution of the puzzle comes from a careful treatment of the boundary conditions satisfied by the "wave-function" $\tilde{z}$ in (2.2). At first glance this sounds very surprising. Indeed, in case of D3-branes we argued that exactly the same solution that solves (2.2) without D3-branes will still solve (2.2) after D3-branes are added. Obviously the same solution has the same boundary conditions. This makes a lot of sense: the boundary condition for $\tilde{z}$ should always be the same - coupling $\beta$ in (2.3) should remain zero while vev $\alpha$ can vary. The subtlety we are missing is due to $\tau_{0}$ in (2.3) which can change what we call $\beta$. In AdS/CFT the boundary conditions are to be formulated at a holographic screen located at some large physical radius $r_{\text {cutoff }}$ which morally corresponds to some physical UV cut-off in field theory. The radial variable $\tau$ at the same time is dimensionless, related to $r$ though the conifold deformation parameter $\varepsilon$

$$
\tau=\log \left(r^{3} / \varepsilon^{2}\right)+O\left(r^{-1}\right) .
$$

Since $\varepsilon$ is the only dimensionful parameter of the solution, no dimensionless quantity can depend on it and accordingly $\varepsilon$ is commonly taken to be one. But this situation changes if one considered two holographic backgrounds, the original and the perturbed one. Say, we start with the KS background and put the holographic screen at $r_{\text {cutoff }}$. Based on the asymptotic behavior of $\tilde{z}$ at large $r$

$$
z=\alpha\left(\log \left(r^{3} / \varepsilon^{2}\right)-1\right)+\beta
$$

where $\alpha$ and $\beta$ are arbitrary parameters, and comparing with the known solutions describing baryonic branch [13-15] we conclude that

$$
a:=\left.\frac{d z}{d \log r^{3}}\right|_{r=r_{\text {cutoff }}},
$$

is a vev, while

$$
b:=z(r)-\left.a\left(\log \left(r^{3} / \varepsilon^{2}\right)-1\right)\right|_{r=r_{\text {cutoff }}},
$$

is a coupling of $\hat{U}$. Now, if we consider a slightly perturbed background with new $\varepsilon$ we still want to keep the same boundary conditions at the same radius $r_{\text {cutoff }}$, namely (2.11) to stay zero, while (2.10) can be arbitrary. As one can see the effect of changing $\varepsilon$ will be in shifting the definition of $\beta$.

Let us now return to the puzzling examples discussed above. The pure KS background and the KS background with $p$ D3-branes correspond holographically to different gauge theories which have completely different (in fact totally unrelated) $\varepsilon$. But if $p=\ell M$ for some integer $\ell$ these two backgrounds describe two different vacua of the same theory in which case the new and the old $\varepsilon$ 's are related by $[7,16]$

$$
\varepsilon_{M}^{2}=\varepsilon^{2} e^{\frac{2 \pi \ell}{M}} .
$$


Based on this relation we conjecture that choosing

$$
\varepsilon_{p}^{2}=\varepsilon^{2} e^{\frac{2 \pi p}{M^{2}}}
$$

in presence of any number $p$ of D3-branes will define correct boundary conditions for $\tilde{z}$ by identifying (2.10) with the vev and (2.11) with the coupling of $\hat{U}$ (these formulae implicitly define $\left.\tau_{0}\right)$. Let us emphasize: while (2.12) is a relation of deformation parameters for two distinct solutions (which correspond to different vacua fo the same field theory) and is proven to be correct, (2.13), together with (2.10) and (2.11) is a conjecture about holographic dictionary for the $\tilde{z}$ mode. The test of the backreacted anti-D3-branes solution advertised in the introduction should be understood as a check of this conjecture as well.

It becomes clear now why supergravity approximation did not "see" the mass $m_{U}^{2}$ in case D3-branes were added. Indeed the same GHK mode with the same $\alpha, \beta, \tau_{0}$ solves the supergravity equations of motion. But since $\varepsilon$ is different, for $\alpha, \beta$ to have the same interpretation in terms of vev and coupling, $\tau_{0}$ should now change. Hence the same GHK solution has different interpretation now: it corresponds to the mode with some non-trivial vev $U$ and some non-zero coupling of $\hat{U}$. This solution describes not only D3-branes but also perturbation of theory by $\hat{U}$ ! In fact there is another solution of (2.2) with the nonzero eigenvalue $m_{U}^{2}$ and vanishing (2.11). This is the correct solution describing motion along the baryonic branch when the only new ingredient is a stack of D3-branes.

The supergravity solutions describing infinitesimal perturbation of KS by gaugino masses also have new value of $\varepsilon$. As a result to find corresponding eigenvalue $m_{U}^{2}$ one has to solve $(2.2)$ with the new potential $V_{z}$ and the new boundary conditions, requiring (2.11) to be zero. As we will show later a small miracle happens and the effect of the new potential cancels against the effect of the new boundary conditions such that there is a zero mode solution for any $m_{i}$, confirming that $m_{U}^{2}$ remains zero, as expected.

\subsection{The baryonic branch uplift from supergravity}

Now we are ready to calculate the appropriate equation for the "wave-function" $\tilde{z}$ of the $U$ mode - the generalization of (2.2) for the more general backgrounds. First, we notice there is only one $\mathbb{Z}_{2}$-odd linearized mode which has the appropriate UV asymptotic corresponding to dimension 2 and correspondingly we have to find the equations describing fluctuation of this mode (and all other modes it couples to) in some abstract $\mathbb{Z}_{2}$-even background. Such equations were derived in [22] for the Klebanov-Strassler background, with the purpose of studying the mass spectrum of certain scalar glueballs. Here we will generalize this result to a full $\mathbb{Z}_{2}$-even $\mathrm{SU}(2) \times \mathrm{SU}(2)$ symmetric ansatz (colloquially called the PT-ansatz after Papadopoulos and Tseytlin [23]). The only equation we need here is (the details can be found in appendix A): ${ }^{3}$

$$
\tilde{z}^{\prime \prime}-V_{\tilde{z}} \tilde{z}=-m^{2}\left[e^{-2(A+4 p)} \tilde{z}+\frac{3 \sqrt{6} P}{4} e^{-A-4 p-x-\frac{\Phi}{2}}\left(f^{\prime} e^{-y}+k^{\prime} e^{y}\right) \tilde{\omega}\right]
$$

\footnotetext{
${ }^{3} \mathrm{~A}$ similar result was previously obtained by C. Ahn and T. Tesileanu.
} 
where $V_{\tilde{z}}$ is the following function of the PT scalars (in Einstein frame):

$$
\begin{aligned}
V_{\tilde{z}}= & \frac{1}{2} \cosh (2 y)+\frac{1}{2} e^{-4 x}\left[2 e^{-12 p}-e^{4 x}+e^{2 x-2 y+\Phi}\left(e^{2 y}(2 P-F)+F\right)^{2}\right] \\
& -\frac{1}{2} e^{-2(x+y)-\Phi}\left(f^{\prime}+e^{2 y} k^{\prime}\right)^{2}-4\left(A^{\prime}+p^{\prime}\right)^{2}+y^{\prime 2}
\end{aligned}
$$

Here $m^{2}$ is the mass of the baryonic branch parameter $U$, it's different from $m_{U}^{2}$ only by some normalization factor.

Above we have introduced

$$
\tilde{z}=4 z e^{2 A+2 p}, \quad \tilde{\omega}=\frac{\sqrt{2}}{3 P \sqrt{3}} \sigma^{\prime} e^{A+10 p+3 x-\Phi / 2},
$$

using the original GHK wave-functions $z$ and $\sigma^{\prime}$ from $[13,14,22]$, also see (A.2), (A.8). When restricted to the KS solution with $\varepsilon$ taken to be one, $V_{\tilde{z}}$ reduces to

$$
V_{\tilde{z}}^{K S}=2 \sinh ^{2} y_{0}=\frac{2}{\sinh ^{2} \tau},
$$

and the massless equation is solved by the GHK mode

$$
\tilde{z}_{0}=\tau \operatorname{coth} \tau-1
$$

To compute the mass for a general linear perturbation around the Klebanov-Strassler background, we expand the potential $V_{z}$ as

$$
V_{\tilde{z}}=V_{\tilde{z}}^{0}+\delta V_{\tilde{z}}
$$

and take $V_{\tilde{z}}^{0}=V_{\tilde{z}}^{K S}$. We use the 0 subscript for the modes of the unperturbed solution. Since the mass of the $U$ mode vanishes at the zeroth-order, we don't need to compute linear fluctuations of the mode $\tilde{\omega}$, and as a consequence the equation for $\tilde{z}$ decouples.

We begin by multiplying both sides of $(2.14)$ by $\tilde{z}_{0}$ and integrate from 0 to $\infty$ :

$$
\int_{0}^{\infty}\left[\tilde{z}_{0} \tilde{z}^{\prime \prime}-V_{\tilde{z}}^{0} \tilde{z}_{0} \tilde{z}-\delta V_{\tilde{z}} \tilde{z}_{0}^{2}\right] d \tau=-m^{2} \kappa_{0}
$$

where $\kappa_{0}$ is the coefficient of $m^{2}$ in (2.14) evaluated at the zeroth-order:

$$
\kappa_{0}=\int_{0}^{\infty}\left[e^{-2\left(A_{0}+4 p_{0}\right)} \tilde{z}_{0}^{2}+\frac{3 \sqrt{6} P}{4} e^{-A_{0}-4 p_{0}-x_{0}}\left(f_{0}^{\prime} e^{-y_{0}}+k_{0}^{\prime} e^{y_{0}}\right) \tilde{z}_{0} \tilde{\omega}_{0}\right] d \tau .
$$

This constant can be evaluated numerically (see below). We observe that from (2.14) we have at the zeroth-order

$$
\frac{2}{\sinh ^{2} \tau} \tilde{z}_{0}=\tilde{z}_{0}^{\prime \prime}
$$

so we can integrate by parts the first two terms in the left-hand-side, to obtain

$$
\int_{0}^{\infty}\left[\tilde{z}_{0} \tilde{z}^{\prime \prime}-\frac{2}{\sinh ^{2} \tau} \tilde{z}_{0} \tilde{z}\right] d \tau=\int_{0}^{\infty}\left[\tilde{z}_{0} \tilde{z}^{\prime \prime}-\tilde{z}_{0}^{\prime \prime} \tilde{z}\right] d \tau=\left[\tilde{z}_{0} \tilde{z}^{\prime}-\tilde{z}_{0}^{\prime} \tilde{z}\right]_{0}^{\infty}
$$


Note that the UV asymptotic of the original wave-function (2.18) is $\tilde{z}_{0} \sim \tau-1$, while for the perturbed solution $\tilde{z}$ we allow for a general behavior

$$
\tilde{z} \sim \tau+\delta \tau-1
$$

Here the shift in $\tau$ coordinate $\delta \tau$ is the same as new $\tau_{0}=1-\delta \tau$. As we explained in section 2.2 it is related to the shift in the deformation parameter of the conifold $\varepsilon$ for the given background in question. Noticing that the IR limit does not contribute we find

$$
\left[\tilde{z}_{0} \tilde{z}^{\prime}-\tilde{z}_{0}^{\prime} \tilde{z}\right]_{0}^{\infty}=-\delta \tau
$$

The mass formula we obtain is thus:

$$
\delta \tau+\int_{0}^{\infty} \delta V_{\tilde{z}} \tilde{z}_{0}^{2} d \tau=m^{2} \kappa_{0} .
$$

We now discuss how to compute various quantities that enter the formula above. First, we would like to determine the shift $\delta \tau$, which is done by matching running of D5-charge in the UV (compare with (2.3))

$$
Q^{D 5}(r)=\mathrm{a} \tau+\mathrm{b}
$$

The idea behind the calculation of $\delta \tau$ is the following. One requires that two solutions which correspond to different states in the same theory have the same charge $Q^{D 5}$ at some large radius $r_{\text {cutoff }}{ }^{4}$ Through $\tau=\log \left(r^{3} / \varepsilon^{2}\right)$ change of $\varepsilon$ introduces the shift $\tau \rightarrow \tau+\delta \tau$ which in turn should be compensated by the change of $b$ [7]. In the notations introduced in $[4,24]$ linear perturbation of the D5-charge at infinity is controlled by only one mode $\tilde{\phi}_{5}$ which approaches a constant at infinity

$$
Q^{D 5}(\tau) \sim P(\tau-1)+\tilde{\phi}_{5}(\infty)
$$

Hence equivalence of $Q^{D 5}\left(r_{\text {cutoff }}\right)$ for two backgrounds (the original and the perturbed one) takes the form

$$
P\left(\tau_{\text {cutoff }}-1\right)=P\left(\tau_{\text {cutoff }}+\delta \tau-1\right)+\tilde{\phi}_{5}(\infty)
$$

From here we get

$$
\delta \tau=-\frac{\tilde{\phi}_{5}(\infty)}{P} .
$$

So the shift in $\tau$ can be unambiguously computed for the solutions of interest, such as perturbation by the gaugino masses, or backreaction of anti-D3-branes.

Second, we obtain the expression for $\kappa_{0}$ numerically

$$
\kappa_{0}=\int_{0}^{\infty}\left[\frac{h_{0}}{6} \frac{\sinh ^{2} \tau}{(\cosh \tau \sinh \tau-\tau)^{2 / 3}} \tilde{z}_{0}^{2}-\frac{6 P^{2}}{\sinh \tau}(\cosh \tau \sinh \tau-\tau)^{1 / 3} \tilde{z}_{0} \tilde{\omega}_{0}\right] \approx 171.583 P^{2} .
$$

\footnotetext{
${ }^{4}$ Strictly speaking the KPV state described by backreaction of $p$ anti-D3-branes is a state in $\mathrm{SU}(N+$ $M+(M-p)) \times \mathrm{SU}(N+(M-p))$ theory. Therefore to calculate $\delta \tau$ for that solution we would need to guess which $\delta \tau$ is caused by adding $M-p$ D3-branes to the KS background. This is exactly what we did in (2.13).
} 
Third, by expanding (2.15) up to linear order we find that $\delta V_{\tilde{z}}$ can be expressed in terms of the perturbation modes $\tilde{\xi}^{a}$ and $\tilde{\phi}^{a}$ introduced in [24] as follows:

$$
\begin{aligned}
\delta V_{\tilde{z}}= & 2 \sinh \left(2 y_{0}\right) \tilde{\phi}_{2}+e^{-4\left(A_{0}+p_{0}\right)}\left[4 \sinh y_{0} \tilde{\xi}_{2}+\frac{2}{3} e^{-6 p_{0}-2 x_{0}}\left(2 \tilde{\xi}_{1}+\tilde{\xi}_{3}+\tilde{\xi}_{4}\right)\right. \\
& \left.-8 \cosh y_{0}\left(e^{y_{0}} P-F_{0} \sinh y_{0}\right) \tilde{\xi}_{5}-8 \sinh y_{0}\left(e^{y_{0}} P-F_{0} \sinh y_{0}\right) \tilde{\xi}_{6}\right] .
\end{aligned}
$$

This form is instrumental since we can now use the explicit numerical solution of [9] to evaluate the above expression. Given a particular perturbation of KS, formulae (2.30), (2.31), and (2.32) allow us to compute the baryonic branch uplift (the mass of the particle associated with the motion along the branch). In the next section we will perform such a calculation for various solutions of interest.

\section{Gaugino mass perturbation}

In this section we discuss the Klebanov-Strassler field theory perturbed by infinitesimally small gaugino masses which softly break supersymmetry. As was mentioned before there are two pairs of gauginos and hence two masses $m_{1}, m_{2}$. Therefore on gravity side we expect two linearized solutions. One gravity solution of this kind was obtained in [21]. It corresponds to some (unspecified) linear combination of $m_{1}, m_{2}$. The second linearly independent solution was obtained in $[7,9]$.

In what follows we use the notations of [9] for both solutions. Thus the two independent parameters of the linearized gravity solutions are denoted by $X_{2}$ and $X_{7}$ that are related to $m_{1}, m_{2}$ by an unspecified linear transformation. The supersymmetry breaking modes $\tilde{\xi}^{a}$ of the solutions in question are known analytically

$$
\begin{aligned}
\tilde{\xi}_{1}= & \tilde{\xi}_{3}=\tilde{\xi}_{4}=0 \\
\tilde{\xi}_{2}= & \frac{X_{2}}{4} \operatorname{csch}^{3}(\tau)(\sinh (2 \tau)-2 \tau)^{2} \\
& \quad-P X_{7}\left[\operatorname{csch}(\tau)+\tau\left(\cosh (\tau)-2 \operatorname{coth}(\tau) \operatorname{csch}(\tau)+\tau \operatorname{csch}^{3}(\tau)\right)\right], \\
\tilde{\xi}_{5}= & \frac{X_{2}}{P}-\frac{3 X_{7}}{2} \\
\tilde{\xi}_{6}= & \frac{X_{2}}{P} \tau \operatorname{csch}(\tau)+X_{7}\left[-\frac{1}{2} \cosh (\tau)-\tau \operatorname{csch}(\tau)\right] \\
\tilde{\xi}_{7}= & \frac{X_{2}}{P}[\tau \operatorname{coth}(\tau)-1] \operatorname{csch}(\tau) \\
& +X_{7}\left[\operatorname{csch}(\tau)-\tau \operatorname{coth}(\tau) \operatorname{csch}(\tau)+\frac{1}{2} \sinh (\tau)\right] \\
\tilde{\xi}_{8}= & -\left(X_{2}-P X_{7}\right)\left[\operatorname{coth}(\tau)-\tau-2 \tau \operatorname{csch}^{2}(\tau)+\tau^{2} \operatorname{coth}(\tau) \operatorname{csch}^{2}(\tau)\right]
\end{aligned}
$$

The solution of [21] corresponds to the subset $X_{2}=P X_{7} \cdot{ }^{5}$ The modes $\tilde{\phi}^{a}$ are given by integral expressions which can be evaluated numerically. In the following we will need the

\footnotetext{
${ }^{5}$ The relation with the notation of [7] is: $X_{2}^{\text {here }}=2 X_{2}^{\text {there }}, X_{7}^{\text {here }}=-2 X_{7}^{\text {there }}$.
} 
UV behavior of one particular mode $\tilde{\phi}_{5}$, which corresponds to first-order perturbation of the PT scalar $f$ and which enters in the UV behavior of D5 charge (2.28). From [9] we have

$$
\tilde{\phi}_{5}=\tilde{\phi}_{5}(\infty)+\mathcal{O}\left(r^{-1}\right)
$$

where (see for example eq. (93) of [9] with $X_{1}=0$ )

$$
\tilde{\phi}_{5}(\infty) \approx-19.5477 P\left(2 X_{2}+P X_{7}\right) .
$$

We also need the expression for the mode $\tilde{\phi}_{2}$ (perturbation of the metric mode $y$ ):

$$
\tilde{\phi}_{2}=-\frac{32}{\sinh \tau} \int \frac{\sinh u \tilde{\xi}_{2}(u)}{(\cosh u \sinh u-u)^{2 / 3}} d u,
$$

for which we could not find an analytic expression. We now compute the relevant integrals that enter the mass formula derived in the previous section. First we would need the explicit expression for $\delta V_{\tilde{z}}$. By plugging (3.1) into (2.32) we obtain

$$
\begin{aligned}
\delta V_{\tilde{z}}= & -\frac{4 \cosh \tau}{\sinh ^{2} \tau} \tilde{\phi}_{2}+\frac{8 P}{\sinh ^{4} \tau(\cosh \tau \sinh \tau-\tau)^{2 / 3}}\left[24 \tau^{2}-6+4 \cosh (2 \tau)+2 \cosh (4 \tau)\right. \\
& -26 \tau \sinh (2 \tau)+\tau \sinh (4 \tau)] X_{7}-\frac{192(\cosh \tau \sinh \tau-\tau)^{4 / 3}}{\sinh ^{4} \tau} X_{2} .
\end{aligned}
$$

Next, we evaluate the integral from (2.26) numerically

$$
\int_{0}^{\infty} \delta V_{\tilde{z}} \tilde{z}_{0}^{2} d \tau \approx-19.5477\left(2 X_{2}+P X_{7}\right) \text {. }
$$

At this point we recognize that the result is precisely the value of $\tilde{\phi}^{5}(\infty) / P$ found in (3.3). Thus we have checked numerically that a small miracle happens and the contribution of $\delta V_{\tilde{z}}$ cancels the contribution from the new boundary conditions leaving the baryonic branch flat at the origin, in agreement with the field theory analysis

$$
m_{U}^{2}=\frac{1}{P \kappa_{0}}\left(P \delta \tau+\tilde{\phi}_{5}(\infty)\right)=0 .
$$

In fact the field theory suggests that the baryonic branch will remain flat at linear order in $m_{i}$ everywhere along the branch, not only at the origin. That simply means that every solution from the BGMPZ family should admit two linearized perturbations which change leading UV boundary conditions only for relevant dimension 3 operators (masses of gauginos). In other words linearized solutions found in [7, 9, 21] should exist for any value of $U$. It would be very interesting to develop a technique similar to [24] to systematically study linearized perturbations around any SUSY background and find the linearized solutions dual to gaugino mass perturbations for any value of $U$ explicitly.

Another interesting question is what happens with the baryonic branch beyond the linear order in $m_{i} \cdot{ }^{6}$ There is no reason to believe the branch will remain flat anymore as one would expect contributions of the sort

$$
m_{U}^{2} \sim\left|m_{i}\right|^{2}+O\left(m_{i}^{3}\right) .
$$

\footnotetext{
${ }^{6}$ This question was raised by I. Klebanov.
} 
The sign of the emerging mass $m_{U}^{2}$ is not clear. If it is positive that would mean the locus of the baryonic branch remains as a stable vacuum in the theory. Otherwise the theory exhibits a run-away behavior. To understand in field theory which scenario would take place is a difficult problem because of non-holomorphic nature of the $\left|m_{i}^{2}\right|$ term. At the same time the answer can be obtained on the gravity side, by first constructing the background dual to the gaugino mass perturbation beyond linear order (this can be done either in perturbation theory in $m_{i}$ or numerically) and then using the machinery of section 2.3.

\section{Uplift of baryonic branch by (anti)D3-branes}

We now turn to the uplifting by D3 or anti-D3 branes. We first perform the computation of the emergent mass in the closed string channel by applying the method described in the previous sections. We then look at the same mass from the open string perspective, and compare the results. For simplicity in this section we will set $P=1$.

\subsection{Uplift from supergravity}

In this subsection we compute the mass $m_{U}^{2}$ (up to some universal coefficient), once D3 or anti-D3 branes are added to the KS background.

We begin with the uplifting of the baryonic branch in the presence of mobile D3-branes. While D3 branes are not BPS objects on the baryonic branch, they become BPS at the origin $U=0$. This greatly simplifies the mass formula (2.26), since as was advertised in section $2.2, \delta V_{\tilde{z}}$ vanishes for a regular supersymmetric perturbation. The emergent mass is then given simply by the shift in $\tau$ coordinate:

$$
\delta \tau^{D 3}=m_{D 3}^{2} \kappa_{0} .
$$

Using (2.8) and (2.13) the shift $\delta \tau$ can be easily calculated to be:

$$
\delta \tau^{D 3}=\frac{\pi}{8} p .
$$

Accordingly, the result for the mass $m_{D 3}^{2}$ at the linear order in $p$ is

$$
m_{D 3}^{2}=\frac{\delta \tau^{D 3}}{\kappa_{0}} \approx 0.00228868 p .
$$

We now turn to the uplift by anti-D3 branes. The solution corresponding to linearized backreaction of anti-D3 branes on the Klebanov-Strassler geometry has been formally obtained in terms of integrals in $[4,7]$ and was simplified and fully evaluated numerically in [9]. In what follows we will rely heavily on the numerical results of [9]. More generally, we will compute the mass for almost general non-supersymmetric perturbation of the deformed conifold, preserving the $\mathrm{SU}(2) \times \mathrm{SU}(2) \times \mathbb{Z}_{2}$ symmetries of the KS background. Such perturbations are parametrized by integration constants denoted $X_{1}, \ldots X_{8}$. We then impose the anti-D3 boundary conditions discussed in [7, 9] to fix all the integration constants in terms of the number $p$ of anti-D3 branes and hence get the emergent mass in case of perturbation by the anti-D3-branes. 
We first need to evaluate the integral of $\delta V_{\tilde{z}}$ in (2.26). The numerical procedure is quite involved but straightforward yielding

$$
\int_{0}^{\infty} \delta V_{\tilde{z}} \tilde{z}_{0}^{2} d \tau \approx-48.6843 X_{1}-39.0955 X_{2}+22.6041 X_{4}-11.9448 X_{6}-19.5477 X_{7} .
$$

We are setting $X_{3}=0$ since this integration constant corresponds to a highly divergent UV perturbation not induced by (anti)D3-branes presence. Note that when $X_{1}=X_{4}=X_{6}=0$ we recover the value for the gaugino mass solution (3.5). By enforcing the anti-D3 boundary conditions described in eq. (74) of [9], we get the following result

$$
\int_{0}^{\infty} \delta V_{\tilde{z}} \tilde{z}_{0}^{2} d \tau \approx 2.98167 p
$$

We would also need the boundary term $\delta \tau$, which is given by the value of the perturbation mode $\tilde{\phi}_{5}$ at infinity. The general expression is found by expanding in the UV the integral form of [9], which results in

$$
\phi_{5}(\infty) \approx 64.9006 X_{1}-39.0955 X_{2}+16.525 X_{4}+0.213416 X_{6}-19.5477 X_{7}-36.4747 X_{8} .
$$

For anti-D3-brane perturbation one finds:

$$
\tilde{\phi}_{5}(\infty) \approx 2.21552 p \text {. }
$$

Combining all contributions together, we arrive at the final result for the anti-D3 branes:

$$
\delta \tau+\int_{0}^{\infty} \delta V_{\tilde{z}} \tilde{z}_{0}^{2} d \tau \approx 0.766142 p .
$$

Eventually we find

$$
m_{\bar{D} 3}^{2} \approx 0.00446514 p
$$

The masses $m_{D 3}^{2}, m_{\overline{D 3}}^{2}$ we found from supergravity are related to $m_{U}^{2}$ defined in field theory by a normalization coefficient which depends on the definition of $\hat{U}$. To get rid of this dependence we can consider the ratio

$$
\frac{m_{D 3}^{2}}{m_{\bar{D} 3}^{2}} \approx 0.512567 .
$$

In the next section, we will compute the same ratio using probe approximation for D3 and anti-D3-branes.

\subsection{Probe computation of the induced masses}

Now we would like to repeat the calculation of mass $m_{U}^{2}$ in presence of D3 and anti-D3branes in the open string channel. This calculation consists of two parts. First part is to calculate the potential along the baryonic branch $V(U)$ emerging due to the presence of the branes. At linear order in the number of branes this calculation is rather trivial and was done in [16]. For $p$ D3-branes the potential is (see (15.2) of [16])

$$
V_{D 3}(U)=p \frac{T_{3}}{\gamma} \frac{U^{2}}{e^{-\Phi(0)}+1},
$$


where $\gamma$ is some constant, while for $p$ anti-D3 it is (see (14.10) of [16])

$$
V_{\bar{D} 3}(U)=p \frac{T_{3}}{\gamma} \frac{U^{2}}{e^{-\Phi(0)}-1} .
$$

Here $\Phi(0)$ is the $U$-dependent value of dilaton at the tip $\tau=0$.

Second part would be to calculate the kinetic term $K(U)$ associated with the motion along the baryonic branch (in other words $K(U)$ is a metric on the moduli space $d s^{2}=$ $K(U) d U^{2}$ ). At the zeroth order in $p$ this calculation does not depend on $p$ and only depends on peculiarities of the BGMPZ solutions (or GHK mode for $K(0)$ ). Once $K$ is known the mass would be given by the usual $m_{U}^{2}=V^{\prime \prime}(0) / 2 K(0)$.

A general method of calculating $K$ was put forward in [25-27]. It would be an interesting exercise to calculate $K(0)$ (and more generally $K(U)$ ) to match $m_{D 3}^{2}, m_{\bar{D} 3}^{2}$ found in the previous subsection. In this paper we prefer to avoid calculating $K$ by considering the ratio $m_{D 3}^{2} / m_{\bar{D} 3}^{2}=V_{D 3}^{\prime \prime}(0) / V_{\bar{D} 3}^{\prime \prime}(0)$ which is $K$-independent.

Using the small $U$ expansion for dilaton (see (13.14) of [16])

$$
\phi(\tau=0, U)=-2^{-11 / 3} U^{2} I(0)+\lambda U^{4}+\mathcal{O}\left(U^{6}\right)
$$

(here $I(0) \approx 0.718050$ is the value of the KS warp-factor at the tip and $\lambda$ is some numerical constant), the desired mass ratio can be found to be

$$
\frac{m_{\bar{D} 3}^{2}}{m_{D 3}^{2}}=\frac{2^{25 / 3} \lambda}{I(0)^{2}}-1
$$

In order to calculate $\lambda$ we need to express the dilaton at the tip in terms of $U$. A general formula expressing the dilaton through other fields is given by (52) of [28]. Upon expanding near $\tau=0$ one finds

$$
e^{4 \Phi(0)}=-72 \frac{(1-2 \xi)^{3}}{U^{3}}
$$

Here

$$
\xi(U)=\frac{1}{2}+\frac{U}{42^{2 / 3}}-\frac{I_{0} U^{3}}{246^{2 / 3}}+\frac{\mu}{2} U^{5}+\mathcal{O}\left(U^{7}\right)
$$

is an IR parameter that parametrizes solutions from the BGMPZ family (we apologize for possible clash of notations with $\tilde{\xi}_{a}$ from section 3 ). Combining all together we arrive at

$$
\frac{m_{\bar{D} 3}^{2}}{m_{D 3}^{2}}=\mu \frac{2^{1 / 3} 3^{2 / 3} 384}{I(0)^{2}}-\frac{7}{3} .
$$

The unknown constant $\mu$ defined in (4.16) is to be calculated numerically. A detailed account of this calculation can be found in the appendix B, while here we just state the final result

$$
\mu \approx 0.00219499 \text {. }
$$

With this value the desired mass ratio turns out to be

$$
\left.\frac{m_{D 3}^{2}}{m_{\bar{D} 3}^{2}}\right|_{\text {probe }}=\left(\mu \frac{2^{1 / 3} 3^{2 / 3} 384}{I(0)^{2}}-\frac{7}{3}\right)^{-1} \approx 0.512567
$$


The result of the probe computation is in a full agreement with the supergravity result (4.10). This confirms that our conjecture (2.13) together with the interpretation of boundary conditions (2.10), (2.11) is correct. Furthermore this means the linearized mode describing backreaction of anti-D3-branes on the conifold (gravity background dual to the KPV state) passes this comprehensive test with the flying colors.

\section{Discussion}

In this paper we calculated the uplift of the baryonic branch (the mass of the scalar associated with the motion along the baryonic branch) in case the KS background is modified by some $\mathbb{Z}_{2}$-even perturbation. Some of our results hold for perturbation of any magnitude, but we mostly focus on linear effects due to infinitesimally small gaugino masses and presence of D3 or anti-D3-branes. In case of gaugino masses we found, in agreement with the field theory expectation, that the baryonic branch remains flat i.e. the corresponding scalar is massless. An interesting question for the future would be to understand what happens with the baryonic branch beyond the linear order in gaugino masses.

The rest of the paper deals with the baryonic branch uplift whenever D3 or anti-D3branes are present. The latter case is very interesting because it provides a way to probe recently found linearized solution describing backreaction of anti-D3-branes placed at the tip of the deformed conifold. Our calculation shows that this solution passes an elaborate test and reproduces the emergent mass calculated using probe approximation precisely. This is a very encouraging confirmation that the boundary conditions and the resulting linearized solution formulated and found in $[4,7,9]$ is correct.

Now we would like to discuss what this means for the status of this solution, in particular if our finding provides any new evidence proving existence of the meta-stable KPV state or reliability of the dS compactification scenario of String Theory.

First of all we would like to emphasize that the original argument of [2] concerns the regime $g_{s} p \ll 1$ i.e. when the number of anti-D3-branes $p$ is small. A more subtle question is if the meta-stability continues for $g_{s} p \gg 1$ while $p \ll M$ and if the corresponding state admits a dual supergravity description. A number of arguments based on the standard curvature estimate $R^{4} \sim\left(g_{s} p\right) \alpha^{\prime 2}$ and comparison with the related case of Polchinski and Strassler [29] suggest that there should be such a supergravity background, but the necessary complexity of the corresponding solution (in particular angular dependence) has hampered all attempts to construct it so far. Moreover, in an effort to bypass this complexity, a search for a KPV-like polarization channel for the fully backreacted but smeared anti-D3 solution has been recently performed in [30] albeit with negative results. Another issue regarding the smeared solution which has been recently investigated is the presence of singularities in three-form fluxes. ${ }^{7}$

Unfortunately our analysis can not contribute in a definite way to the problem of existence of the self-consistent fully nonlinear holographic background dual to the KPV state when $g_{s} p \gg 1$ and $p \ll M$. The reason is the linear nature of the tested solution. This should be understood in the following way. Let us consider for example, a field

\footnotetext{
${ }^{7}$ See for example [31-34] for an account of the vivid debate on the interpretation of such singularities.
} 
theoretic configuration describing a dense star. So far the star has not collapsed into a black hole the density of matter is finite everywhere and one expects to find a smooth classical configuration of fields (including gravity) describing it. There is no need to introduce any singularity, like the one in the center of the Schwarzschild solution, which strictly speaking make sense only in quantum gravity. However at the linear level one oversees the interaction (gravitational and all other) of the matter forming the star and hence know nothing about its spatial distribution. Looking from afar, all matter sits in the same point and the resulting Newtonian (electrical etc.) potential $\varphi=G M_{\mathrm{star}} / r$ is clearly singular. But certainly this is not a sign of any inconsistency. Moreover linear solution correctly describes leading interaction between the star and other objects. At the same time correct linear solution obviously does not guarantee that the matter composing the star is capable of resisting the gravitational collapse and that the corresponding smooth field configuration exists. This can only be checked if one goes into full non-linear regime and either finds the corresponding smooth solution or shows that is not possible.

The case of anti-D3-branes is very similar. In this paper we checked that the linear solution is indeed correct, but that is simply not enough to say anything about full non-linear regime. Moreover the case of backreacting anti-D3-branes is more complicated than the collapsing star (which is spherically symmetric) because it is expected to break geometrical symmetries of the problem due to emerging special $\mathbb{S}^{2}$ with NS5-brane wrapped around it.

\section{Acknowledgments}

We would like to thank C. Ahn, I. Klebanov, T. Tesileanu, and B. Safdi for collaboration on the early stages of this project and to M. Graña, Z. Komargodski, L. McAllister, and N. Seiberg for discussions. AD would like to thank International Institute of Physics (Federal University of Rio Grande do Norte) for hospitality while part of this work was done. AD gratefully acknowledges support from a Starting Grant of the European Research Council (ERC STG grant 279617) and the grant RFBR 12-01-00482. The work of SM is supported by a Contrat de Formation par la Recherche of CEA/Saclay, by the ERC Starting Independent Researcher Grant 259133 - ObservableString and by the ERC Advanced Grant "Strings and Gravity" (Grant.No. 32004).

\section{A Derivation of $V_{\tilde{z}}$}

We derive the equation of motion (2.14) for the mode $\tilde{z}$ by following the procedure of [22], namely by linearizing ten dimensional Einstein equations. Instead of giving full details of this derivation, we show here a quick way, mentioned in $[13,14]$, to derive the potential $V_{\tilde{z}}$ in (2.15) from the one-dimensional effective action of Papadopoulos and Tseytlin (PT) [23]. The metric of the ansatz is

$$
d s_{10}^{2}=e^{2 A+2 p-x} d s_{1,3}^{2}+e^{-6 p-x} d \tau^{2}+\left(e^{x+g}+a^{2} e^{x-g}\right)\left(e_{1}^{2}+e_{2}^{2}\right)+e^{x-g}\left(\tilde{\epsilon}_{1}^{2}+\tilde{\epsilon}_{2}^{2}\right)+e^{-6 p-x} \tilde{\epsilon}_{3}^{2} .
$$


This metric preserves the $\mathbb{Z}_{2}$ symmetry which interchanges the two $S^{2}$ s only if $e^{g}+a^{2} e^{-g}=$ $e^{-g}$. Since we are interested in an expansion around the $\mathbb{Z}_{2}$ symmetric solution we define

$$
\begin{aligned}
e^{g} & =\frac{1}{\cosh y-c z}, \\
a & =\frac{\sinh y}{\cosh y-c z},
\end{aligned}
$$

where $c$ is a $\mathbb{Z}_{2}$-breaking parameter. Next, we derive an effective action for $z$ at the $c^{2}$ order. We note that the field $\chi$ enters only through its derivative, so we eliminate it using the EOM

$$
\chi^{\prime}=e^{-y} z\left(f^{\prime}+e^{2 y} k^{\prime}\right) .
$$

The effective action then takes the form

$$
\begin{aligned}
\mathcal{L}_{z}= & \frac{z^{2} e^{4 A-8 p-4 x-2 y-\Phi}}{4\left(1+e^{2 y}\right)^{2}}\left[2 e^{2(6 p+x+y+\Phi)}\left(e^{4 y}-1\right) P F+e^{2(6 p+x)}\left(e^{2 y}+1\right) f^{\prime 2}\right. \\
& +e^{2(6 p+x+y)}\left(e^{2 y}+1\right)^{2} f^{\prime} k^{\prime}+e^{2(6 p+x+2 y)+2 y}\left(e^{2 y}+1\right) k^{\prime 2} \\
& \left.-e^{2 y+\Phi}\left(2\left(e^{2 y}+1\right)\left(1+e^{2 y}-2 e^{6 p+2 x+y}+2 e^{12 p+2 x+2 y+\Phi} P^{2}\right)+e^{4(3 p+x)}\left(e^{2 y}-1\right)^{2} y^{\prime 2}\right)\right] \\
& -\frac{1}{4} e^{4 A+4 p} z^{\prime}\left(z^{\prime}-2 z y^{\prime} \tanh y\right) .
\end{aligned}
$$

Now we define

$$
\tilde{z}=4 z e^{2 A+2 p}
$$

so that the equation of motion for $\tilde{z}$ derived from (A.5) is

$$
\tilde{z}^{\prime \prime}-V_{\tilde{z}} \tilde{z}=0,
$$

with $V_{\tilde{z}}$ given by (2.15). One can check that this equation agrees with the one obtained from Einstein equations when $m^{2}=0$. To get the full equation (2.14) when $m^{2} \neq 0$ one needs to generalize the PT Ansatz by allowing dependence of all fields on the external coordinates and adding a linear perturbation:

$$
\delta B_{2}=(\chi-\sigma) d g^{5}-\sigma^{\prime} d \tau \wedge g^{5},
$$

and then solving directly the equations of motion, following the procedure in [22].

\section{B Particulars of numerical integration}

In this section we describe the numerical procedure we used to compute the parameter $\mu$ in (4.16). We use a shooting technique to numerically evaluate two particular modes of the BGMPZ family of solutions, $a(\tau)$ and $v(\tau)=e^{6 p+2 x}$. They are determined by a coupled system of ODEs [15]:

$$
a^{\prime}=-\frac{a \sinh \tau(\tau+a \sinh \tau)}{\tau \cosh \tau-\sinh \tau}-\frac{1}{v}\left[\sqrt{-1-a^{2}-2 a \cosh \tau}(1+a \cosh \tau) \operatorname{csch} \tau\right],
$$




$$
\begin{aligned}
v^{\prime}= & -\frac{3 a \sinh \tau}{\sqrt{-1-a^{2}-2 a \cosh \tau}} \\
& +v\left[-a^{2} \cosh ^{3} \tau+2 a \tau \operatorname{coth} \tau+a \cosh ^{2} \tau(2-4 \tau \operatorname{coth} \tau)+\tau \operatorname{csch} \tau\right. \\
& \left.+\cosh \tau\left(1+2 a^{2}-\left(2+a^{2}\right) \tau \operatorname{coth} \tau\right)\right] /\left[\left(1+a^{2}+2 a \cosh \tau\right)(\tau \cosh \tau-\sinh \tau)\right] .
\end{aligned}
$$

Since it is much easer to get perturbative series expansion in the IR rather than in the UV, we start by computing Taylor series for the functions $a(\tau)$ and $v(\tau)$ for small $\tau$, up to a large number of terms, by solving the system (B.1). The coefficients are polynomial functions of the parameter $\xi$ :

$$
a(\tau, \xi)=-1+\sum_{l=1}^{l^{\star}} a_{l}(\xi) \tau^{l}+\mathcal{O}\left(\tau^{l^{\star}+1}\right), \quad v(\tau, \xi)=\sum_{l=1}^{l^{\star}} v_{l}(\xi) \tau^{l}+\mathcal{O}\left(\tau^{l^{\star}+1}\right) .
$$

We can easily compute the series for up to $l^{\star}=30$; the first terms being

$$
\begin{aligned}
& a(\tau)=-1+\xi \tau^{2}+\frac{1}{60}\left(-3+29 \xi-114 \xi^{2}+36 \xi^{3}\right) \tau^{4}+\mathcal{O}\left(\tau^{6}\right), \\
& v(\tau)=t+\frac{1}{120}\left(5-84 \xi+84 \xi^{2}\right) \tau^{3}+\mathcal{O}\left(\tau^{5}\right) .
\end{aligned}
$$

These series approximate the functions $a(\tau)$ and $v(\tau)$ with an extremely high precision in the IR. Next, we choose a particular value of $\xi$ close to $\xi=1 / 2$ (that corresponds to $U=0$ ), which we call $\xi^{\star}$ and we calculate $a\left(\tau_{I R}\right)$ and $v\left(\tau_{I R}\right)$ for some particular $\tau_{I R}$ using (B.2). The value of $\tau_{I R}$ is chosen such that $\tau_{I R}$ is sufficiently smaller than 1 and series (B.2) converges rapidly. For example $\tau_{I R}=1 / 10$. The values of $a, v$ at $\tau_{I R}$ are used as boundary conditions to numerically evaluate with Mathematica NDSolve the ODEs (B.1) from $\tau_{I R}$ to a point $\tau_{U V}$ in the moderate UV region. Lastly, we need to determine the value of $U$ from the UV series of $a(\tau)$. By solving (B.1) we get UV series up to sixth-order. The first terms are given below

$$
\begin{aligned}
& a(\tau)=-2 e^{-\tau}+U e^{-5 \tau / 3}(-1+\tau)-\frac{1}{2} U^{2} e^{-7 \tau / 3}(-1+\tau)^{2}+\mathcal{O}\left(e^{-3 \tau}\right), \\
& v(\tau)=\frac{3}{2}+\frac{9}{16} U^{2} e^{-4 \tau / 3}\left(6-4 \tau+\tau^{2}\right)+e^{-2 \tau}(\mathrm{c}-6 \tau)+\mathcal{O}\left(e^{-8 \tau / 3}\right) .
\end{aligned}
$$

The constant c which first enters at order $e^{-2 \tau}$ in the expansion of $v$ should be thought as a function of $U$. With these UV expansions we numerically find $U^{\star}$, associated with $\xi^{\star}$, by matching the series with the result of NDSolve in $\tau_{U V}$. Finally using the expansion (4.16) we determine $\mu$ though

$$
\xi^{\star}=\frac{1}{2}+\frac{U^{\star}}{42^{2 / 3}}-\frac{I_{0}\left(U^{\star}\right)^{3}}{246^{2 / 3}}+\frac{\mu}{2}\left(U^{\star}\right)^{5}+\ldots
$$

Using different values of $\xi^{\star}=\left(\frac{1}{2}-5 \cdot 10^{-4} ; \frac{1}{2}-10^{-4}\right)$ and playing with the value of $\tau_{U V}=$ 15,20 and $\tau_{I R}=1 / 10$ we find $\mu \approx 0.00219499(4)$, with the precision of about $10^{-6}$.

Open Access. This article is distributed under the terms of the Creative Commons Attribution License (CC-BY 4.0), which permits any use, distribution and reproduction in any medium, provided the original author(s) and source are credited. 


\section{References}

[1] I.R. Klebanov and M.J. Strassler, Supergravity and a confining gauge theory: duality cascades and $\chi S B$ resolution of naked singularities, JHEP 08 (2000) 052 [hep-th/0007191] [INSPIRE].

[2] S. Kachru, J. Pearson and H.L. Verlinde, Brane/flux annihilation and the string dual of a nonsupersymmetric field theory, JHEP 06 (2002) 021 [hep-th/0112197] [INSPIRE].

[3] S. Kachru, R. Kallosh, A.D. Linde and S.P. Trivedi, De Sitter vacua in string theory, Phys. Rev. D 68 (2003) 046005 [hep-th/0301240] [INSPIRE].

[4] I. Bena, M. Graña and N. Halmagyi, On the existence of meta-stable vacua in Klebanov-Strassler, JHEP 09 (2010) 087 [arXiv:0912.3519] [INSPIRE].

[5] H.-Y. Chen, Y. Nakayama and G. Shiu, On D3-brane dynamics at strong warping, Int. J. Mod. Phys. A 25 (2010) 2493 [arXiv:0905.4463] [InSPIRE].

[6] O. DeWolfe, S. Kachru and M. Mulligan, A gravity dual of metastable dynamical supersymmetry breaking, Phys. Rev. D 77 (2008) 065011 [arXiv:0801.1520] [INSPIRE].

[7] A. Dymarsky, On gravity dual of a metastable vacuum in Klebanov-Strassler theory, JHEP 05 (2011) 053 [arXiv: 1102.1734] [INSPIRE].

[8] I. Bena, G. Giecold, M. Graña, N. Halmagyi and S. Massai, On metastable vacua and the warped deformed conifold: analytic results, Class. Quant. Grav. 30 (2013) 015003 [arXiv:1102.2403] [INSPIRE].

[9] I. Bena, G. Giecold, M. Graña, N. Halmagyi and S. Massai, The backreaction of anti-D3 branes on the Klebanov-Strassler geometry, JHEP 06 (2013) 060 [arXiv:1106.6165] [INSPIRE].

[10] S. Kachru et al., Towards inflation in string theory, JCAP 10 (2003) 013 [hep-th/0308055] [INSPIRE].

[11] I. Bena, G. Giecold, M. Graña and N. Halmagyi, On the inflaton potential from antibranes in warped throats, JHEP 07 (2012) 140 [arXiv:1011.2626] [INSPIRE].

[12] O. Aharony, A note on the holographic interpretation of string theory backgrounds with varying flux, JHEP 03 (2001) 012 [hep-th/0101013] [INSPIRE].

[13] S.S. Gubser, C.P. Herzog and I.R. Klebanov, Symmetry breaking and axionic strings in the warped deformed conifold, JHEP 09 (2004) 036 [hep-th/0405282] [INSPIRE].

[14] S.S. Gubser, C.P. Herzog and I.R. Klebanov, Variations on the warped deformed conifold, Comptes Rendus Physique 5 (2004) 1031 [hep-th/0409186] [INSPIRE].

[15] A. Butti, M. Graña, R. Minasian, M. Petrini and A. Zaffaroni, The baryonic branch of Klebanov-Strassler solution: a supersymmetric family of $\mathrm{SU}(3)$ structure backgrounds, JHEP 03 (2005) 069 [hep-th/0412187] [INSPIRE].

[16] A. Dymarsky, I.R. Klebanov and N. Seiberg, On the moduli space of the cascading $\mathrm{SU}(M+p) \times \mathrm{SU}(P)$ gauge theory, JHEP 01 (2006) 155 [hep-th/0511254] [INSPIRE].

[17] I.R. Klebanov and E. Witten, Superconformal field theory on three-branes at a Calabi-Yau singularity, Nucl. Phys. B 536 (1998) 199 [hep-th/9807080] [INSPIRE].

[18] I.R. Klebanov and E. Witten, AdS/CFT correspondence and symmetry breaking, Nucl. Phys. B 556 (1999) 89 [hep-th/9905104] [INSPIRE]. 
[19] A. Dymarsky and S. Kuperstein, Non-supersymmetric conifold, JHEP 08 (2012) 033 [arXiv:1111.1731] [INSPIRE].

[20] S.B. Giddings, S. Kachru and J. Polchinski, Hierarchies from fluxes in string compactifications, Phys. Rev. D 66 (2002) 106006 [hep-th/0105097] [INSPIRE].

[21] S. Kuperstein and J. Sonnenschein, Analytic nonsupersymmtric background dual of a confining gauge theory and the corresponding plane wave theory of hadrons, JHEP 02 (2004) 015 [hep-th/0309011] [INSPIRE].

[22] M.K. Benna, A. Dymarsky, I.R. Klebanov and A. Solovyov, On normal modes of a warped throat, JHEP 06 (2008) 070 [arXiv:0712.4404] [INSPIRE].

[23] G. Papadopoulos and A.A. Tseytlin, Complex geometry of conifolds and five-brane wrapped on two sphere, Class. Quant. Grav. 18 (2001) 1333 [hep-th/0012034] [INSPIRE].

[24] V. Borokhov and S.S. Gubser, Nonsupersymmetric deformations of the dual of a confining gauge theory, JHEP 05 (2003) 034 [hep-th/0206098] [INSPIRE].

[25] G. Shiu, G. Torroba, B. Underwood and M.R. Douglas, Dynamics of warped flux compactifications, JHEP 06 (2008) 024 [arXiv:0803.3068] [INSPIRE].

[26] M.R. Douglas and G. Torroba, Kinetic terms in warped compactifications, JHEP 05 (2009) 013 [arXiv: 0805.3700] [inSPIRE].

[27] A.R. Frey, G. Torroba, B. Underwood and M.R. Douglas, The universal Kähler modulus in warped compactifications, JHEP 01 (2009) 036 [arXiv:0810.5768] [INSPIRE].

[28] A. Dymarsky, Flavor brane on the baryonic branch of moduli space, JHEP 03 (2010) 067 [arXiv:0909.3083] [INSPIRE].

[29] J. Polchinski and M.J. Strassler, The string dual of a confining four-dimensional gauge theory, hep-th/0003136 [INSPIRE].

[30] I. Bena, M. Graña, S. Kuperstein and S. Massai, Polchinski-Strassler does not uplift Klebanov-Strassler, JHEP 09 (2013) 142 [arXiv:1212.4828] [INSPIRE].

[31] I. Bena et al., Persistent anti-brane singularities, JHEP 10 (2012) 078 [arXiv:1205.1798] [INSPIRE].

[32] I. Bena, M. Graña, S. Kuperstein and S. Massai, Anti-D3 branes: singular to the bitter end, Phys. Rev. D 87 (2013) 106010 [arXiv:1206.6369] [InSPIRE].

[33] I. Bena, A. Buchel and O.J.C. Dias, Horizons cannot save the landscape, Phys. Rev. D 87 (2013) 063012 [arXiv:1212.5162] [INSPIRE].

[34] I. Bena, J. Blaback, U.H. Danielsson and T. Van Riet, Antibranes cannot become black, Phys. Rev. D 87 (2013) 104023 [arXiv: 1301.7071] [InSPIRE]. 